Purdue University Purdue e-Pubs

International Compressor Engineering Conference

School of Mechanical Engineering

2010

\title{
Fluid Flow in a Screw Pump Oil Supply System for Reciprocating Compressors
}

Marcus Vinicius C. Alves

Federal University of Santa Catarina

Jader R. Barbosa

Federal University of Santa Catarina

Alvaro T. Prata

Federal University of Santa Catarina

Fernando A Ribas

Embraco

Follow this and additional works at: https://docs.lib.purdue.edu/icec

Alves, Marcus Vinicius C.; Barbosa, Jader R.; Prata, Alvaro T.; and Ribas, Fernando A, "Fluid Flow in a Screw Pump Oil Supply System for Reciprocating Compressors" (2010). International Compressor Engineering Conference. Paper 1998.

https://docs.lib.purdue.edu/icec/1998

This document has been made available through Purdue e-Pubs, a service of the Purdue University Libraries. Please contact epubs@purdue.edu for additional information.

Complete proceedings may be acquired in print and on CD-ROM directly from the Ray W. Herrick Laboratories at https://engineering.purdue.edu/ Herrick/Events/orderlit.html 


\title{
Fluid Flow in a Screw Pump Oil Supply System for Reciprocating Compressors
}

\author{
Marcus V.C. ALVES ${ }^{1}$, Jader R. BARBOSA, Jr. ${ }^{1 *}$, Alvaro T. PRATA ${ }^{1}$, Fernando A. RIBAS, Jr. ${ }^{2}$ \\ ${ }^{1}$ Polo - Research Laboratories for Emerging Technologies in Cooling and Thermophysics \\ Federal University of Santa Catarina, Department of Mechanical Engineering \\ Florianopolis, SC, 88040-900, Brazil \\ ${ }^{2}$ Embraco Compressors \\ Joinville, SC, 89219-901, Brazil \\ * Corresponding Author (Phone/Fax: +55 48 32345166, E-mail: jrb@polo.ufsc.br)
}

\begin{abstract}
This work presents an analysis of an oil supply system for reciprocating compressors. The system is based on a single screw pump attached to the bottom end of the vertical rotating shaft immersed in the oil sump. The fluid flow in the pump was modeled with a semi-analytical approach based on the solution for the laminar fully developed oil flow in a screw extruder. The screw pump model is coupled with that for the flow in the shaft region so as to provide an estimate of the oil flow rate and of the so-called 'climbing-time', i.e., the amount of time needed for a fluid particle to travel from the oil sump to the top of the shaft. The calculation method was verified against experimental data and CFD modeling results.
\end{abstract}

\section{INTRODUCTION}

Lubrication is a major issue in compressor design because it is directly related to mechanical losses, wear and reliability. In addition to sufficiently large oil flow rates, one requires that the lubricant oil becomes immediately available to the hydrodynamic bearings and to the piston-cylinder gap immediately after the motor start-up (Prata and Barbosa, 2009). Since the geometry of oil supply systems is generally too complex for local measurements of the oil flow parameters, one frequently resorts to numerical modeling of the fluid flow in the pump, shaft and bearings in order to obtain specific parameters of the oil flow in such systems.

A number of computational fluid dynamics (CFD) studies of oil supply in hermetic compressors have been carried out in the open literature. Bernardi (2000), Cho et al. (2002), Cui (2004) and Lückmann et al. (2009) have investigated different types of supply systems under transient and steady-state conditions in rotary and reciprocating compressors using commercial CFD software. Over time, these models have become increasingly more detailed and realistic with respect to the pumping system geometry used in the simulations. However, they still rely on a number of simplifying assumptions such as the absence of refrigerant dissolved in oil, isothermal conditions, constant physical properties etc. Although the simplifying assumptions help to keep the computational time at a tolerable level, this can still be considered very large and thus prohibitive for design and optimization purposes.

The oil supply system investigated in the present work is shown schematically in Fig. 1. As pointed out by Lückmann et al. (2009), the pump design must be simple and efficient and, in some hermetic compressors, use is made of the actual rotation of the shaft as the driving force to overcome gravity and friction as oil is forced through the channels and passages to feed the shaft bearings (Fig. 1.a). The lower part of the supply system, which is partially immersed in the oil sump, consists of a single screw extruder with a fixed center pin and an external rotating barrel (Fig. 1.b). Viscous shear is the driving force for pumping the oil. After leaving the screw pump at the lower part of the system, the oil enters the shaft, where it flows through a helical groove machined on its outer 
surface and feeds the shaft bearings. From the top of the shaft, the oil is expelled to the internal crankcase environment and falls back to the sump in its lower part.

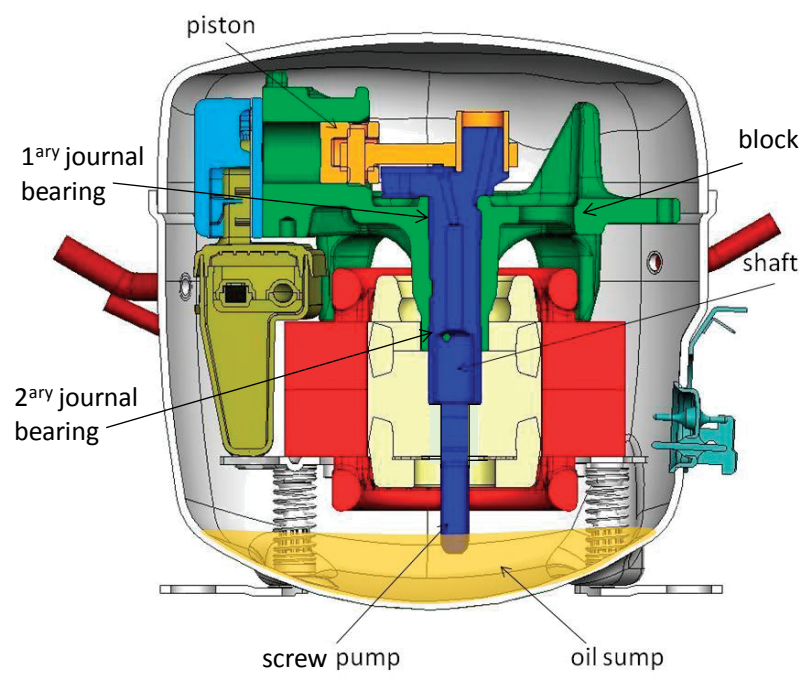

(a)

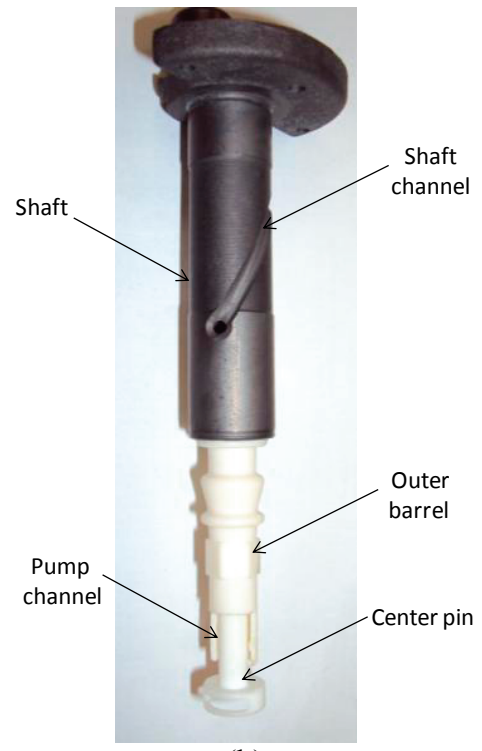

(b)

Figure 1: Geometry of oil pumping system. (a) Compressor with pump. (b) Screw pump oil supply system.

The purpose of the present paper is to advance a semi-analytical model for predicting the oil flow rate in the screw pump system described above. The Generalized Integral Transform Technique (GITT) (Cotta, 1993) has been used to determine analytically the down-channel component of the velocity field in the lower and upper parts of the supply system. The pumped oil flow rate is calculated numerically based on the individual relationships for the down-channel flow rates in the screw pump and shaft regions. The results are compared with experimental data and numerical predictions obtained via computational fluid dynamics (CFD) (Alves, 2007). An important result of the present analysis is the so-called 'climbing-time', i.e., the amount of time needed for a fluid particle to travel from the oil sump to the top of the shaft. This is of particularly significance as a means of avoiding the contact between the sliding parts which may result in excessive friction and wear.

\section{MODELING}

\subsection{Down-Channel Flow Rate}

The kernel of the calculation method is the classical 2D model for the laminar fully developed flow in a single screw extruder of Rowell and Finlayson (1922, 1928). The model has been extended by Li and Hsieh (1996) to take into account the effect of the motion of the screw flights on the down-channel velocity field. More recently, the resulting non-homogeneous boundary value problem of Li and Hsieh has been solved analytically via the GITT by Alves et al. (2009) for a wide range of values of the screw channel aspect ratio.

The basic geometry of the screw pump in the lower part is shown in Fig. 2(a) and consists of a fixed center pin and a rotating outer barrel which incorporates the screw flights. The outer barrel is attached to the shaft. In the shaft region shown in Fig. 2(b), the oil flows through a helical groove machined on the shaft and the outer barrel (i.e., the cylinder block) is held stationary. The intermediate region that connects the lower and upper parts plays no role in the pumping mechanism except for connecting the pump to the shaft. For this reason, in the model, the volume of this piece of geometry can be neglected, and the outlet of the screw pump becomes the inlet of the shaft channel. 


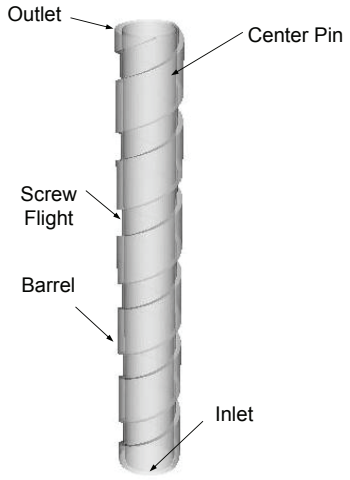

(a)

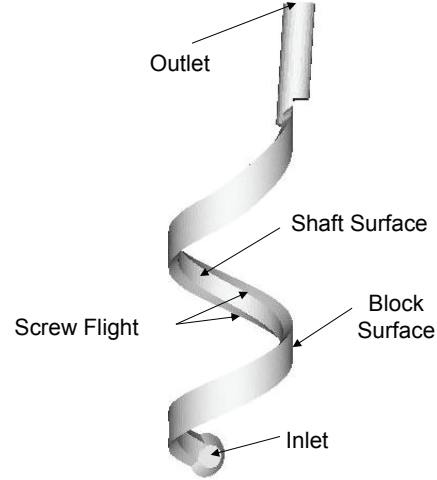

(b)

Figure 2. Geometry of the (a) screw pump and (b) shaft channel regions.

In both domains (pump and shaft), the screw curvature is assumed to be small and the curved surfaces of the channel can be unwrapped to become flat plates (see Figs. 3 and 4 for the main parameters and systems of coordinates). The flow is assumed to be laminar, fully developed, isothermal and incompressible. In dimensionless form (Alves et al., 2009), the momentum balance equation for the velocity component in the $z$ direction is given by,

$$
\frac{\partial^{2} w^{*}}{\partial x^{* 2}}+\frac{\partial^{2} w^{*}}{\partial y^{* 2}}=\frac{H^{2}}{\mu \Omega R_{B}} \frac{d p}{d z}+\frac{\rho g H^{2} \sin (\beta)}{\mu \Omega R_{B}}=k=\text { constant }
$$

where,

$$
\begin{aligned}
x^{*} & =\frac{x}{H} \\
y^{*} & =\frac{y}{H} \\
w^{*} & =\frac{w}{\Omega R_{B}}
\end{aligned}
$$

$g$ is the acceleration due to gravity, $\rho$ is the oil density, $\beta$ is the helix angle, $\mu$ is the oil viscosity, $H$ is the height of the helix channel, $\Omega$ is the angular velocity of the shaft and $R_{B}$ is the barrel radius. The boundary conditions for the screw pump region are given by,

$$
\begin{gathered}
w^{*}\left(x^{*}, 1\right)=\cos (\beta) \\
w^{*}\left(x^{*}, 0\right)=0 \\
w^{*}\left(\gamma, y^{*}\right)=\left[1+\kappa\left(y^{*}-1\right)\right] \cos (\beta) \\
w^{*}\left(0, y^{*}\right)=\left[1+\kappa\left(y^{*}-1\right)\right] \cos (\beta)
\end{gathered}
$$

where $\kappa$ is the screw curvature radius. Conversely, for the shaft region, the boundary conditions are as follows,

$$
\begin{gathered}
\tilde{w}^{*}\left(\tilde{x}^{*}, 1\right)=0 \\
\tilde{w}^{*}\left(\tilde{x}^{*}, 0\right)=(1-\tilde{\kappa}) \cos (\tilde{\beta}) \\
\tilde{w}^{*}\left(\tilde{\gamma}, \tilde{y}^{*}\right)=\left[1+\tilde{\kappa}\left(\tilde{y}^{*}-1\right)\right] \cos (\tilde{\beta}) \\
\tilde{w}^{*}\left(0, \tilde{y}^{*}\right)=\left[1+\tilde{\kappa}\left(\tilde{y}^{*}-1\right)\right] \cos (\tilde{\beta})
\end{gathered}
$$


where ' $\sim$ ' stand for the variables associated with the shaft region. The boundary conditions are also illustrated in Figs. 3(c) and 4(b), respectively. As can be seen, the motion of the screw flights is taken into account in the boundary conditions for the two domains, which makes the boundary value problem non-homogeneous.

Equation (1) has been solved with both sets of boundary conditions via the GITT (Alves et al., 2009) and the resulting velocity distributions were integrated over the cross-section area of the screw pump and the shaft channels. The resulting expressions for the screw pump and the shaft channel flow rate are given by,

$$
Q^{*}=-\frac{1}{2}(\kappa-2) \gamma \cos (\tilde{\beta})+\frac{1}{\gamma} \sum_{i=1}^{\infty} \frac{\left[1-(-1)^{i}\right]}{\alpha_{i}^{2}}\left[C_{1}\left(\mathrm{e}^{\alpha_{i}}-1\right)+C_{2}\left(\mathrm{e}^{-\alpha_{i}}-1\right)+\frac{k\left[1-(-1)^{i}\right]}{\alpha_{i}^{3}}\right]
$$

where,

$$
\begin{aligned}
& C_{1}=\left[\frac{k\left(\mathrm{e}^{-\alpha_{i}}-1\right)}{\alpha_{i}^{3}\left(\mathrm{e}^{-\alpha_{i}}-\mathrm{e}^{\alpha_{i}}\right)}-\frac{1}{2} \frac{(\kappa-2) \mathrm{e}^{-\alpha_{i}}}{\alpha_{i}\left(\mathrm{e}^{-\alpha_{i}}-\mathrm{e}^{\alpha_{i}}\right)} \cos (\tilde{\beta})\right]\left[1-(-1)^{i}\right] \\
& C_{2}=\left[\frac{k\left(\mathrm{e}^{\alpha_{i}}-1\right)}{\alpha_{i}^{3}\left(\mathrm{e}^{-\alpha_{i}}-\mathrm{e}^{\alpha_{i}}\right)}+\frac{1}{2} \frac{(\kappa-2) \mathrm{e}^{\alpha_{i}}}{\alpha_{i}\left(\mathrm{e}^{-\alpha_{i}}-\mathrm{e}^{\alpha_{i}}\right)} \cos (\tilde{\beta})\right]\left[1-(-1)^{i}\right]
\end{aligned}
$$

and,

$$
\tilde{Q}^{*}=-\frac{1}{2}(\tilde{\kappa}-2) \tilde{\gamma} \cos (\tilde{\beta})+\frac{2}{\tilde{\gamma}} \sum_{i=1}^{\infty} \frac{\left[1-(-1)^{i}\right]}{\tilde{\alpha}_{i}^{2}}\left[\tilde{C}_{1}\left(\mathrm{e}^{\tilde{\alpha}_{i}}-1\right)+\tilde{C}_{2}\left(\mathrm{e}^{-\tilde{\alpha}_{i}}-1\right)+\frac{\tilde{k}\left[1-(-1)^{i}\right]}{\tilde{\alpha}_{i}^{3}}\right]
$$

where,

$$
\begin{gathered}
\tilde{C}_{1}=-\left[\frac{\tilde{\alpha}_{i}^{2} \cos (\tilde{\beta})-k\left(\mathrm{e}^{-\tilde{\alpha}_{i}}-1\right)}{\tilde{\alpha}_{i}^{3}\left(\mathrm{e}^{-\tilde{\alpha}_{i}}-\mathrm{e}^{\tilde{\alpha}_{i}}\right)}\right]\left[1-(-1)^{i}\right] \\
\tilde{C}_{1}=\left[\frac{\tilde{\alpha}_{i}^{2} \cos (\tilde{\beta})-\tilde{k}\left(\mathrm{e}^{\tilde{\alpha}_{i}}-1\right)}{\tilde{\alpha}_{i}^{3}\left(\mathrm{e}^{-\tilde{\alpha}_{i}}-\mathrm{e}^{\tilde{\alpha}_{i}}\right)}\right]\left[1-(-1)^{i}\right]
\end{gathered}
$$

In the screw pump, because the tip is submerged in the oil sump and the flow is assumed fully developed, the pressure gradient can be approximated by,

$$
\frac{d p}{d z}=-\frac{\rho g h_{\mathrm{oil}}}{L}=-\frac{\rho g h_{\mathrm{oil}}}{h_{B}} \sin (\beta)
$$

where $L$ is the overall length of the pump channel, $h_{\text {oil }}$ is the height of the submerged tip, and $h_{B}$ is the pump height. In the shaft channel region, the pressure gradient is nil since there is no hydrostatic head at the inlet and no back pressure at the outlet. Thus, for the screw pump and shaft channel regions, the value of the constant $k$ in Eq. (1) is given by, 


$$
k=-\frac{H^{2}}{\mu \Omega R_{B}} \frac{\rho g h_{\text {oil }}}{h_{B}} \sin (\beta)+\frac{\rho g H^{2} \sin (\beta)}{\mu \Omega R_{B}}
$$

and,

$$
\tilde{k}=\frac{\rho g \tilde{H}^{2} \sin (\tilde{\beta})}{\mu \Omega \tilde{R}_{B}}
$$

The pumped mass flow rate is obtained via a coupled solution procedure Eqs. (13) and (16) outlined as follows: (i) The flow rate in the screw pump is calculated with Eq. (13) assuming that the shaft channel on top of it is devoid of any oil; (ii) the pressure gradient in the shaft channel which corresponds to this flow rate is determined via the bisection method (Press et al., 1992) using Eq. (16); (iii) this resulting pressure gradient in the shaft channel is incorporated in the new estimate of the flow rate in the screw pump (i.e., it is added to the right hand side of Eq. (20)), which is compared to the flow rate previously calculated in (i). The procedure is repeated until convergence is obtained.

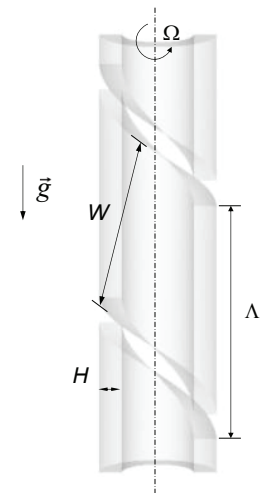

(a)

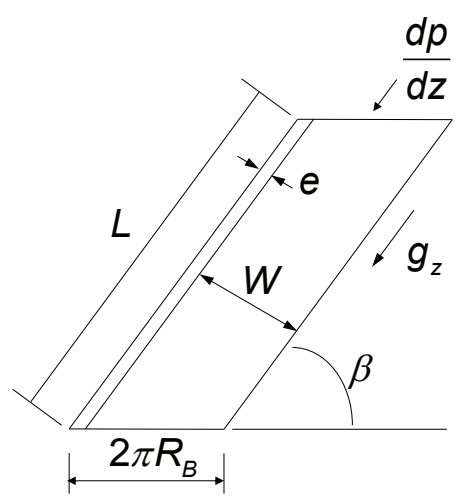

(b)

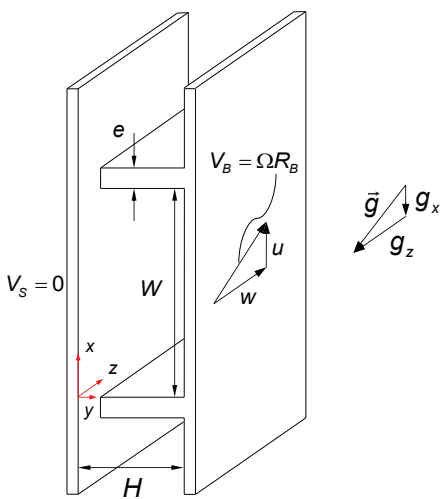

(c)

Figure 3. (a) Basic geometry of the screw pump (a), unwrapped geometry, (c) system of coordinates.

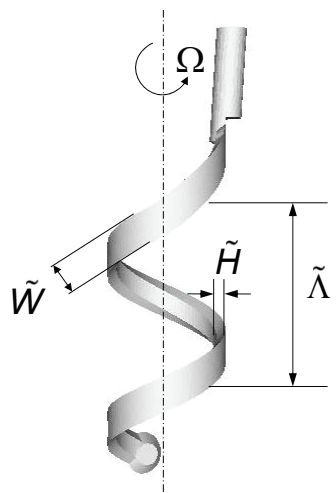

(a)

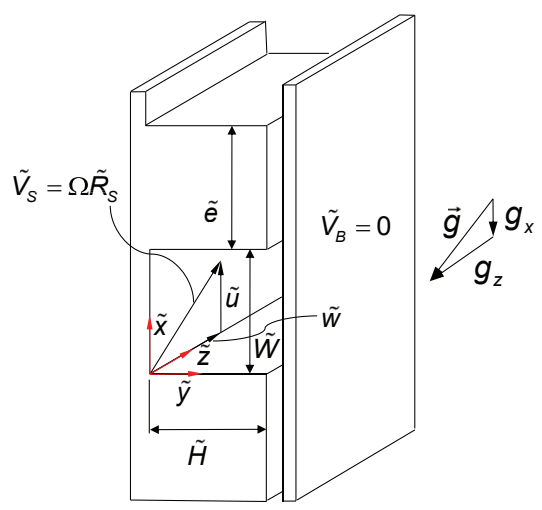

(b)

Figure 4. (a) Basic geometry of the shaft channel, (b) system of coordinates.

\subsection{Climbing Time}

As in Lückmann et al. (2009), the most accurate way to assess the oil climbing time is perhaps to conduct a numerical simulation of the initial transient of the entire oil pumping system via CFD. In the present work, however, given that no transient modeling has been performed, the climbing time is estimated based on the time that a fluid particle would take to go from the bottom to the top of the system in steady-state. The average flow velocity in steady state is given by, 


$$
\bar{V}=\frac{Q}{H W}
$$

where $Q$ is the oil volume flow rate. The total climbing time (screw pump and shaft channel) of a fluid particle can be approximated by,

$$
\Delta t^{*}=\frac{L}{\bar{V}}+\frac{\tilde{L}}{\tilde{\bar{V}}}=\frac{h_{B} H W}{Q \sin (\beta)}+\frac{\tilde{h}_{B} \tilde{H} \tilde{W}}{\tilde{Q} \sin (\tilde{\beta})}
$$

Recognizing that $Q^{*}=\frac{Q}{\omega H^{2} R_{B}}$, and that $Q^{*}=\tilde{Q}^{*}$ (the pump and the shaft channel are connected so the flow rates are equal), one has,

$$
\Delta t^{*}=\frac{1}{Q^{*}}\left[\frac{h_{B} W}{\Omega R_{B} H^{2} \sin (\beta)}+\frac{\tilde{h}_{B} \tilde{W}}{\Omega \tilde{R}_{B} \tilde{H}^{2} \sin (\tilde{\beta})}\right]
$$

\section{RESULTS}

The validity of the models for the flow rate and the climbing time can be assessed by a direct comparison with experimental data and with results obtained via a CFD analysis using commercial software (Fluent, 2006). Figure 5 shows the CFD prediction of the oil volume fraction as a function of time for the screw pump system under the same conditions evaluated in the present paper (Alves, 2007). As can be seen, the oil seems to firstly fill the screw pump almost completely before advancing into the shaft channel. The cross-section of the shaft channel is only partially filled with oil, possibly due to a combination of a larger curvature radius (which yields larger tangential velocities and centrifugal forces acting on the liquid) and a smaller $W / H$ channel aspect ratio in comparison with the screw pump in the lower part.
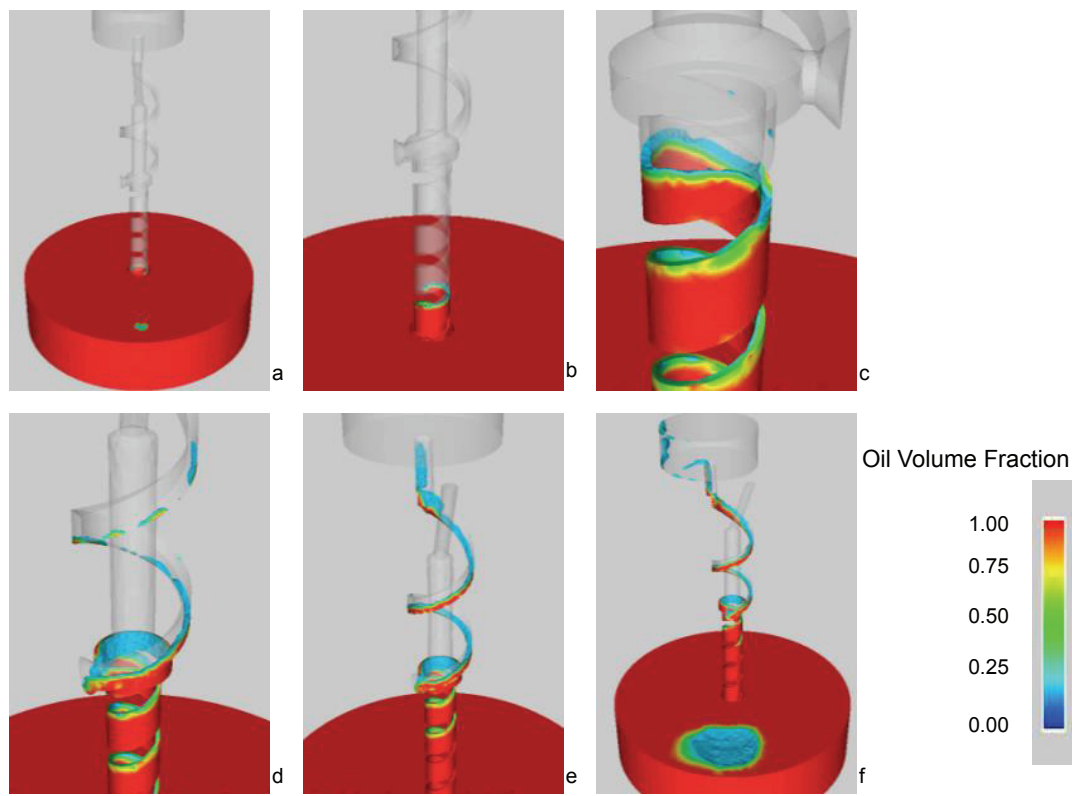

Figure 5. Volumetric oil fraction as function of the dimensionless time.

Dimensionless time: (a) 0.0 ; (b) 1.25 ; (c) 5.67 ; (d) 8.34 ; (e) 10.41 ; (f) 13.75 .

Figure 6 shows the behavior of the dimensionless pumped oil flow rate calculated by means of the semi-analytical model as a function of the shaft angular velocity. The results show a satisfactory agreement with the experimental 
data (maximum error of the order of $+12 \%$ ), which were obtained in a compressor prototype operating at steadystate and under standard conditions for temperature and pressure. Clearly, the main advantage of the semi-analytical method in comparison with the CFD analysis is the computing time; while the former takes only a few seconds to run, the latter took approximately 336 hours in a Pentium D-930 workstation with 2 Gb of RAM.

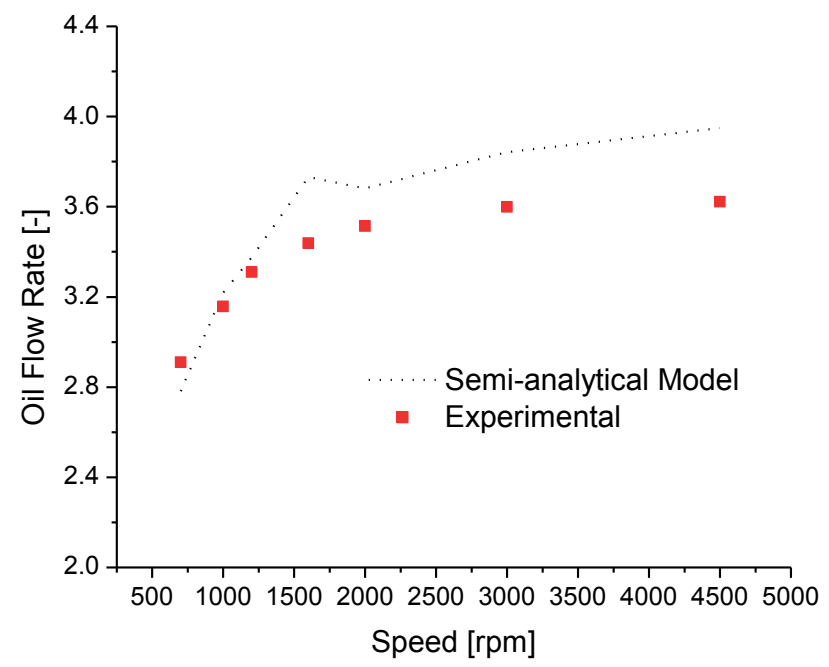

Figure 6. Comparison of the flow rate given by the semi-analytical model with experimental data.

Figure 7 shows the pumped oil flow rate predictions of the CFD model as a function of time for a shaft speed of $1200 \mathrm{rpm}$. A time lag between the flow rates calculated at the pump inlet, shaft channel inlet and shaft channel outlet is clearly observed. The experimental datum for the oil flow rate and the semi-analytical model prediction are also shown in the figure, where the CFD results at steady-state are seen underpredict the experimental datum by approximately $15 \%$. A dimensionless 'climbing time' of approximately 13.75 can be inferred from the CFD results. This agrees well with the value of approximately 17.50 given by the semi-analytical model (Eq. 24). The agreement can be considered satisfactory given the tremendous reduction in the computing time achieved with the semianalytical model.

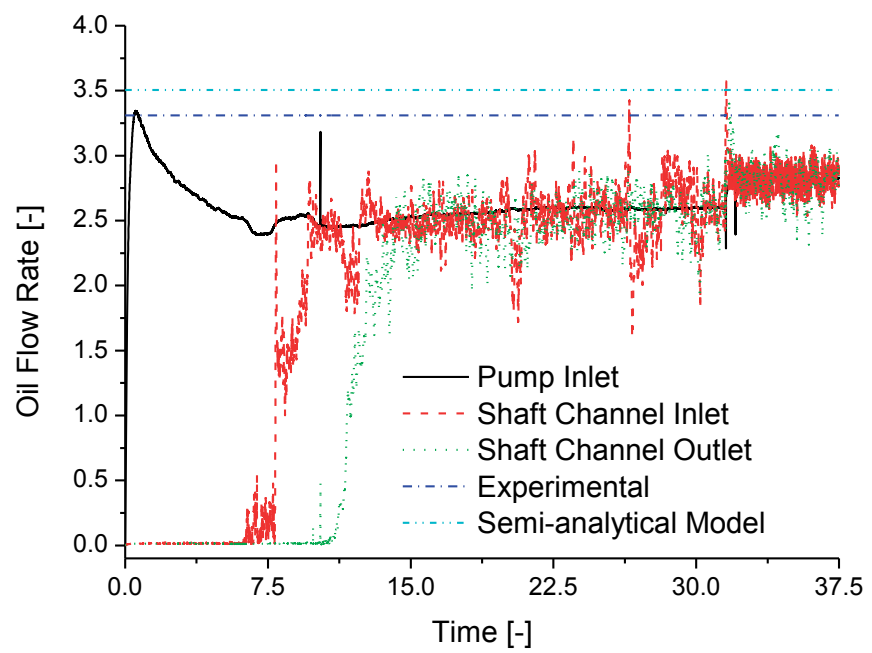

Figure 7. CFD dimensionless oil flow rate predictions as function of time for a shaft angular speed of $1200 \mathrm{rpm}$.

\section{CONCLUSIONS}

An oil pumping system for reciprocating compressors has been described in this paper. The system is based on a single screw extruder attached to the bottom of the shaft which is partially submerged in the oil sump. A model 
based on the analytical solution of the laminar, fully developed oil flow via the GITT has been proposed. The calculated oil flow rates have been compared with experimental data, showing a satisfactory agreement. The predictions of the climbing time were also in accordance with the experimental and CFD modeling results. A remarkable reduction of the computing time for the semi-analytical model in comparison with CFD has been obtained.

\section{NOMENCLATURE}

$\begin{array}{llll}\begin{array}{lll}\text { Roman Symbols } \\ t^{*}=\frac{h_{B}}{\Omega R_{B}} t\end{array} & \text { Dimensionless time } & \Omega & \begin{array}{l}\text { Screw helix angle } \\ \text { Angular velocity }\left(\mathrm{s}^{-1}\right)\end{array} \\ k & \text { Dimensionless pressure drop } & \alpha_{i}=\frac{i \pi}{\gamma} & \begin{array}{l}\text { Eigenvalues } \\ Q^{*}=\frac{Q}{\omega H W R_{B}}\end{array} \\ \bar{V} & \text { Dimensionless flow rate } & \gamma=\frac{W}{H} & \text { Screw aspect ratio } \\ R_{B} & \text { Mean velocity in the channel (m/s) } & \kappa=\frac{H}{R_{B}} & \text { Screw curvature ratio } \\ h_{B} & \text { barrel radius (external radius) (m) } & \tau=\frac{\Lambda}{R_{B}} & \text { Screw helix ratio } \\ \text { Greek Symbols } & \text { Screw height (m) } & \Lambda=2 \pi R_{B} \tan (\beta) & \text { Screw pitch (m) } \\ \mu & & & \end{array}$

\section{REFERENCES}

Alves, M.V.C., 2007, Analysis of oil pumping in hermetic compressors for domestic refrigeration, M.Sc. thesis (in Portuguese), Federal University of Santa Catarina, Brazil.

Alves, M.V.C., Barbosa Jr., J.R., Prata, A.T., 2009, Analytical solution of single screw extrusion applicable to intermediate values of screw channel aspect ratio, J. Food Eng., vol. 92: p. 152-156.

Bernardi, J.D., 2000, CFD simulation of a scroll compressor oil pumping system, Proc. Int. Compressor Engineering Conference at Purdue, p. 707-714.

Cho, H., Yoo, B., Kim, Y., Chung, J.T., 2002, CFD simulation on the oil pump system of a variable speed scroll compressor, Proc. Int. Compressor Engineering Conference at Purdue, Paper C24-1.

Cotta, R.M., 1993, Integral Transforms in Computational Heat and Fluid Flow, CRC Press, Boca Raton, FL.

Cui, M., 2004, Investigation on the oil supply system of a scroll compressor, Proc. Int. Compressor Engineering Conference at Purdue, Paper C93.

FLUENT INC., 2006, Fluent release 6.3.26., Lebanon, NH.

Li, Y., Hsieh, F., 1996, Modeling of flow in a single screw extruder, J. Food Eng., vol. 17: p. 353-375.

Lückmann J.A., Alves, M.V.C., Barbosa Jr., J.R., 2009, Analysis of oil pumping in a reciprocating compressor, Appl. Thermal Eng., vol. 29: p. 3118-3123.

Prata, A.T., Barbosa, Jr. J.R., 2009, Role of the thermodynamics, heat transfer and fluid mechanics of lubricant oil in hermetic reciprocating compressors, Heat Transfer Eng., vol. 30: p. 533-548.

Press, H. W., Teukolsky, S. A., Vetterling, W. T., Flannery, B. P., 2001, Numerical recipes in FORTRAN 77: The art of scientific computing, $2^{\text {nd }}$ Ed., Cambridge University Press, UK.

Rowell, H.S., Finlayson, D., 1922, Screw viscosity pumps, Engineering, vol. 114: p. 606-607.

Rowell, H.S., Finlayson, D., 1928. Screw viscosity pumps, Engineering, vol. 126: p. 249-387.

\section{ACKNOWLEDGEMENTS}

The material presented in this paper is a result of a long-standing technical-scientific partnership between the Federal University of Santa Catarina (UFSC) and Embraco. The authors are indebted to FINEP and CNPq through Grant No. 573581/2008-8 (National Institute of Science and Technology in Refrigeration and Thermophysics). 